\title{
PRIMER REGISTRO DOCUMENTADO DEL SALTEADOR PARDO STERCORARIUS ANTARCTICUS (LESSON, 1831) (STERCORARIIDAE) PARA LAS COSTAS DEL PACÍFICO DESDE EL ARCHIPIÉLAGO DIEGO RAMÍREZ, SUR DE CHILE
}

\author{
FIRST DOCUMENTED RECORD OF BROWN SKUA STERCORARIUS \\ ANTARCTICUS (LESSON, 1831) (STERCORARIIDAE) FOR THE PACIFIC \\ COAST FROM DIEGO RAMIREZ ARCHIPELAGO, SOUTHERN CHILE
}

Victor Raimilla ${ }^{1,2}$

El salteador pardo (Stercorarius antarcticus Lesson, 1831) posee una amplia distribución reproductiva en la zona circumpolar que incluye las mayores islas subantárticas, territorios continentales adyacentes en el sureste de Argentina (costas de Santa Cruz y Chubut) y la península Antártica (Del Hoyo et al. 1996). De esta especie, tres subespecies son reconocidas: S. a. hamiltoni (Hagen, 1952), S. a. antarcticus (Lesson, 1831) y S. a. lonnbergi (Mathews), siendo estas dos últimas probables de ocurrir en las costas del océano Pacífico (Del Hoyo et al. 1996, Ritz et al. 2008). En invierno, las poblaciones de estas subespecies abandonan sus sitios de nidificación y migran a mar abierto (del Hoyo et al. 1996). Desde las Islas South Georgia/Georgias del Sur (54 $4^{\circ} 00^{\prime} \mathrm{S}, 38^{\circ} 03^{\prime} \mathrm{W}$ ) y Falkland/Malvinas $\left(51^{\circ} 43^{\prime} \mathrm{S}, 61^{\circ} 18^{\prime} \mathrm{W}\right)$ se conoce que la subespecie nominal se desplaza invernalmente hacia el sureste de Sudamérica entre los $40^{\circ}$ y $52^{\circ} \mathrm{S}$ asociado a aguas subantárticas del océano Atlántico, mientras que S. a. lonnbergi lo hace más bien hacia aguas más profundas dentro de la plataforma argentina entre los $37^{\circ}$ y $52^{\circ} \mathrm{S}$ (Phillips et al. 2007).
En Chile, la distribución del salteador pardo es aún incierta. Diversos autores indican que la especie utilizaría las costas de Chile continental tras sus desplazamientos invernales desde la Antártica (Araya \& Millie 1986, Couve \& Vidal 2003, Marín 2004, Martínez \& González 2004). Sin embargo, no existen registros que lo confirmen (cf. Reynolds 1935, Bernath 1965, Humphrey et al. 1970, Jehl 1973, Clark 1986, Marín 2004, Imberti 2005). Excepcionalmente, Brito (2001) describe el avistamiento de una pareja el 30 de marzo de 1996 desde las costas de la región de Valparaíso (estero El Yali; $33^{\circ}$ $47^{\prime} S, 71^{\circ} 23^{\prime} \mathrm{W}$; Fig. 1). No obstante, referencias posteriores no consideran este registro (ver Jaramillo 2003, Marín 2004, Martínez \& González 2004), probablemente por la ausencia de una descripción, fotografía, grabación o presencia de una piel que permita confirmar el registro. Recientemente, Cursach et al. (2009) en una revisión sobre las aves de la región del Maule ( $34^{\circ}-36^{\circ} \mathrm{S}$; Fig. 1) mencionan la presencia del salteador pardo para la zona marina regional, sin embargo, no proporcionan mayor información o evidencia del registro.

1 Laboratorio de Ecología, Universidad de Los Lagos. Casilla 933, Osorno, Chile. phalcoboenus@gmail.com

2 Programa de Conservación de Aves Rapaces y Control Biológico. Centro de Estudios Agrarios y Ambientales, Casilla 164, Valdivia, Chile. 
Ante esta ausencia de evidencia, Jaramillo (2003) señala al salteador pardo con estatus indefinido en el Pacífico y como probable de ocurrir sólo en el Paso Drake y frente a la costa Este de Tierra del Fuego en migración post-reproductiva. En la presente nota reportamos el primer registro documentado del salteador pardo para las costas del Pacífico proveniente desde el Archipiélago Diego Ramírez y discutimos sobre su situación para la costa chilena.

Desde el 27 de septiembre al 27 de octubre de 2011 se realizaron trabajos de campo sobre la ecología espacial del albatros de ceja negra (Thalassarche melanophris Temminck) en la Isla Bartolomé (93 ha), Archipiélago Diego Ramírez (56 31' S, $68^{\circ}$ $48^{\prime}$ W), sur de Chile. Este archipiélago se encuentra ubicado aproximadamente a $112 \mathrm{~km}$ al suroeste de Cabo de Hornos y a $700 \mathrm{~km}$ al norte de la Antártica (Schlatter \& Riveros 1997). Importantes colonias de aves marinas se reproducen en este archipiélago: ca. 1.035.000 parejas de petrel azulado (Halobaena caerulea Gmelin), ca. 99.000 parejas de yunco de los canales (Pelecanoides urinatrix Mathews), ca. 55.000 parejas de albatros de ceja negra, ca. 17.000 parejas de albatros de cabeza gris ( $T$. crysostoma Forster), ca. 133.000 parejas de pingüino de penacho amarillo (Eudyptes chrysocome Forster) y ca. 16.000 parejas de pingüino macaroni (E. chrysolophus Brandt) (Lawton et al. 2005, Kirkwood et al. 2007, Robertson et al. 2007). La vegetación del archipiélago está compuesta casi en su totalidad por coirón (Poa flabellata Lam.), la que puede alcanzar hasta los dos metros de altura (Pisano \& Schlatter 1981).

El 29 de septiembre de 2011, a las 15:00 h (horario local) se observó en el lado Oeste de la Isla Bartolomé al primer individuo de salteador pardo posado a menos de $20 \mathrm{~m}$ de una colonia mixta de albatros. El individuo observado se caracterizó por presentar un color pardo cálido uniforme con leves estrías blancas en el cuello y en los flancos,

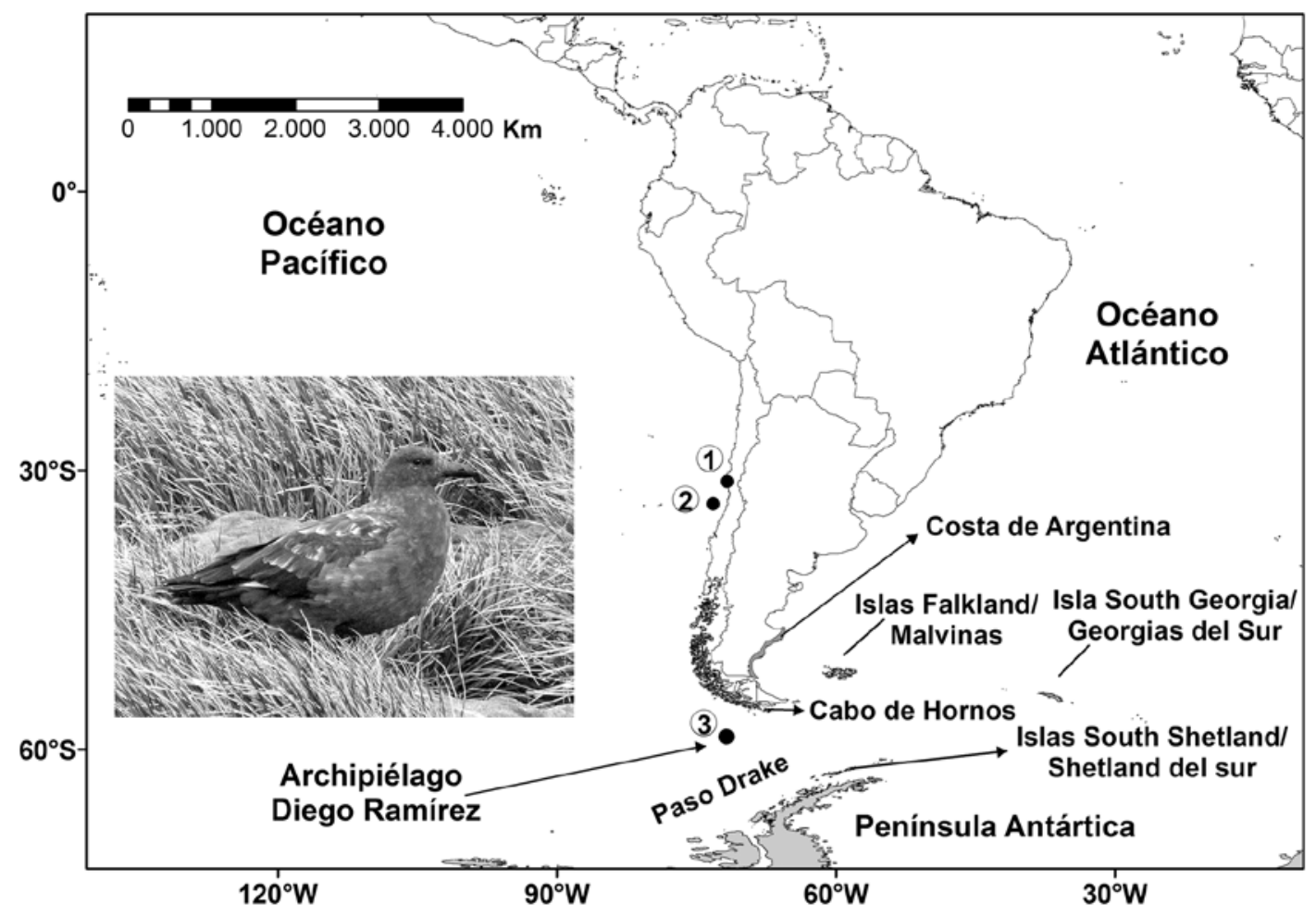

Fig. 1. Registros documentados y no documentados del salteador pardo (Stercorarius antarcticus) para Chile. Los círculos numerados indican la ubicación de los registros: (1) costas de Valparaíso (Brito 1999), (2) mar frente a la región del Maule (Cursach et al. 2009) y (3) Isla Bartolomé, Archipiélago Diego Ramírez (este trabajo). En gris se señalan las colonias de nidificación cercanas (i.e., Islas Falkland/Malvinas, Islas South Georgia/Georgias del sur, Islas South Shetland/Shetland del sur, y costa de Argentina) a la zona del registro documentado en este trabajo (ver fotografía). 
alas oscuras por debajo, contextura relativamente maciza y pico fornido (ver fotografía del ave en Fig. 1). Este patrón de coloración se diferenció del morfo oscuro de salteador polar (S. maccormicki Saunders), el que presenta una coloración dorsal pardo más oscuro con ausencia de manchas blancas y pico relativamente pequeño en comparación al tamaño de la cabeza (Acosta-Hospitaleche et al. 2009, Lowen 2011). Asimismo, la ausencia de corona parda oscura, y de tonos acanelados en el pecho y vientre, permitieron diferenciarlo de un salteador chileno (S. chilensis Bonaparte) (Jaramillo 2003, Lowen 2011).

Entre el 29 de septiembre y el 18 de octubre se observó diariamente a lo menos a un individuo de salteador pardo en la Isla Bartolomé, situación que varió sobre los días 19 y 21 de octubre de 2011 (20 y 22 días después del primer registro, respectivamente) donde se observó en ambas ocasiones a una pareja en vuelo. Estas últimas observaciones ocurrieron entre las 18:30 y 19:00 $\mathrm{h}$, horario donde también se observó un mayor número de salteadores chilenos en vuelo $(N=8)$, probablemente asociado a la salida de las cuevas $e$ inicio de la actividad de vuelo de los petrel azulados (ver Mougeot \& Bretagnolle 2000).

A partir de estos antecedentes se confirma la ocurrencia del salteador pardo para Chile y considerando la ubicación geográfica del Archipiélago Diego Ramírez podría expandirse al paso Drake y límite Este del estrecho de Magallanes coincidiendo con lo sugerido por Jaramillo (2003). Adicionalmente, no se pudo precisar la nidificación mediante evidencia directa (e.g., nidos, huevos o pichones), no obstante, evidencia indirecta como la observación de más de un individuo y el rango de fecha entre registros, el que se encuentra dentro del periodo de arribo de $S$. a. antarcticus a los sitios de nidificación en South Georgia/Georgia del Sur (30 de septiembre al 15 de octubre) y en Islas Falkland/Malvinas (31 de octubre al 24 de noviembre) (Philips et al. 2007), permitirían pensar que la reproducción del salteador pardo en el Archipiélago Diego Ramírez sea posible.

\section{AGRADECIMIENTOS}

Agradezco a Graham Robertson y Cristián G. Suazo, por apoyar mi viaje y estadía en el Archipiélago Diego Ramírez. A la Armada de Chile por permitir el acceso en Isla Bartolomé, y a Ben Wallis y Magnus O'Grady por el transporte vía marítima en el yate "Australis" hasta Bartolomé. Manuel Marín, Ricardo Matus, Pablo Cáceres, Rodrigo Barros y Santiago Imberti colaboraron en la confirmación y comentarios sobre el registro. Sugerencias de Manuel Marín y las de un revisor anónimo mejoraron sustancialmente este trabajo.

\section{LITERATURA CITADA}

Acosta-Hospitaleche, C., D. Montalti \& L.J. Marti. 2009. Skeletal morphoanatomy of the brown skua Stercorarius antarcticus lonnbergi and south polar skua Stercorarius maccormicki. Polar Biology, 32: 759-774.

Araya, B. \& G. Millie 1986. Guía de campo de las aves de Chile. Editorial Universitaria, Santiago, Chile.

Bernath, E.L. 1965. Observations in southern Chile in the southern hemisphere autumn. Auk, 72: 6-13.

Brito, J.L. 2001. Catharacta lonnbergi en el estero del Yali. Boletín Chileno de Ornitología, 8: 36.

Clark, G.S. 1986. Notes on the seabirds observed Turing the Totorore Expedition's Voyage across the Drake Pasaje to the Antarctic Peninsula and return, april 1985. Australasian Seabird Group Newsletter 24: 5-7.

Couve, E. \& C. Vidal. 2000. Birds of Patagonia, Tierra del Fuego and Antarctic peninsula. Editorial Fantástico Sur Birding, Punta Arenas, Chile.

Cursach, J., J. Rau \& C. Suazo. 2009. Sinopsis sobre el conocimiento de las aves en la región del Maule, Chile central. Boletín Chileno de Ornitología, 15: 57-72.

Del Hoyo, J., A. Elliott \& J. Sargatal (eds.). 1996. Handbook of the Birds of the World. Vol. 3: Hoatzin to Auks. Lynx Edicions, Barcelona, España.

Humphrey, P., D. Bridge, P. Reynolds \& R. Peterson 1970. Birds of Isla Grande (Tierra del Fuego). Preliminary Smithsonian Manual Museum of Natural History. University of Kansas, Lawrence.

Imberti, S. 2005. Distribución otoñal de aves marinas y terrestres en canales chilenos. Anales del Instituto de la Patagonia, 33: 21-30. 
Jaramillo, A. 2003. Birds of Chile. Helm Field Guides, Christopher Helm. London.

Jehl, J. Jr. 1973. The distribution of marine birds in chilean waters in winter. Auk, 90: 114-135.

Kirkwood, R., K. Lawton, C. Moreno, J. Valencia, R. Schlatter \& G. Robertson 2007. Estimates of southern rockhopper and macaroni penguin numbers at the Ildefonso and Diego Ramírez Archipelagos, Chile, using quadrat and distance-sampling techniques. Waterbirds, 30: 259-267.

Lawton, K., G. Robertson, R. Kirkwood, J. Valencia, R. Schlatter \& D. Smith 2005. An estimate of popolation sizes of burrowing seabirds at the Diego Ramirez archipelago, Chile, using distance sampling and burrow-scoping. Polar Biology, 29: 229-238.

Lowen, J. 2011. Antarctic wildlife: a visitor's guide to the wildlife of the Antarctic Peninsula, Drake Passage, and Beagle Channel. WildGuides Edicions.

Marín, M. 2004. Lista comentada de las aves de Chile. Lynx Edicions, Barcelona, España.

Martínez, D. \& G. González 2004. Las aves de Chile, nueva guía de campo. Ediciones del Naturalista, Santiago, Chile.

Mougeot, F. \& V. Bretagnolle 2000. Predation risk and moonlight avoidance in nocturnal seabirds. Journal of Avian Biology, 31: 376-387.
Phillips, R.A., P. Catry, J. Silk, S. Bearhop, R. McGill, V. Afanasyev \& I.J. Strange 2007. Movements, winter distribution and activity patterns of Falkland and Brown Skuas: insights from loggers and isotopes. Marine Ecology Progress Series, 345: 281-291.

Pisano, E. \& R.P. Schlatter 1981. Vegetación y flora de las Islas Diego Ramírez (Chile), II comunidades vegetales vasculares. Anales del Instituto de la Patagonia (Chile) 12:195-204.

Reynolds, P.W. 1935. Notes on the birds of Cape Horn. Ibis, 5: 65-101.

Ritz, M.S., C. Millar, G.D. Miller, R.A. Phillips, P. Ryan, V. Sternkopf, D. Liebers-Helbig \& P. Hans-Ulrich 2008. Phylogeography of the southern skua complex-rapid colonization of the southern hemisphere during a glacial period and reticulate evolution. Molecular Phylogenetic and Evolution, 49: 292-303.

Robertson, G., C. Moreno, K. Lawton, J. Arata, J. Valencia \& R. Kirkwood 2007. An estimate of the population sizes of black-browed (Thalassarche melanophrys) and grey-headed (T. chrysostoma) albatrosses breeding in the Diego Ramírez Archipelago, Chile. Emu, 107: 239-244.

Schlatter, R.P. \& G. Riveros 1997. Historia natural del Archipiélago Diego Ramírez, Chile. Serie Científica INACH, 47: 87-112. 